\title{
Research on Application of Array Antenna in United X Band System Calibration
}

\author{
CAI Jin Yao ${ }^{1}, X U$ Rong ${ }^{1}$ \\ 1. China Satellite Maritime Tracking and Control Department, Jiangyin 214431 \\ Caijinyao_52@163.com
}

KeyWords: United X Band system, far-field calibration, array antenna

\begin{abstract}
The distance between the existing calibration tower of the survey ship base and survey ship is only 980 meters which can`t satisfy the United X Band system antenna far-field calibration condition. To solve the problem, a method using array antenna to synthesize quasi plane wave is presented to implement the far field calibration under the condition of the near distance. Sixteen horns were used to build the 4 by 4 array antenna equipment on calibration tower. By controlling the amplitude and phase of the array antenna element, the synthesize wave spreading to the antenna surface created by array antenna is the quasi plane wave which can satisfy the $\mathrm{X}$ band antenna far-field calibration condition. Integrated using optimization theory and genetic algorithm, the weight values of array antenna element amplitude and phase are optimized, thus the synthesize wave can be more closer to the quasi plane wave. The quasi plane wave produced by this method can satisfy the far-field calibration condition in both computer simulation and outfield experiments.
\end{abstract}

\section{Introduction}

As the development of aerospace enterprise, the distance of deep detect become more and more farer, the gain of antenna demand is more and more higher, the aperture become more and more bigger, the frequency is more and more higher. In order to satisfy far-field condition, the calibration distance will be dozens and hundreds of kilometers which can't be realized actually. According to the formula of far-field distance, the far-field distance of 12 meters United X Band antenna is 8160 meters. At the moment, the distance between the existing calibration tower and the survey ship base can't satisfy the United X Band system antenna far-field calibration condition. Previously, we set beacons on the top of high-rise buildings which is located by the survey ship surrounding, thus the way can satisfy the far-field calibration distance condition. However, there are few high-rise buildings behind the survey ship, multipath disturb exist when the height of building is not high enough. Meanwhile, the calibration time spend on the way come and go is quite long, the implementing process and bring equipment is difficult and cumbersome. There are no more effective solutions in the existing literature.

Thus, a method using array antenna to synthesize quasi plane wave is presented. By controlling the amplitude and phase of the array antenna element, the synthesize wave spreading to the antenna surface created by array antenna is the quasi plane wave which can satisfy the $\mathrm{X}$ band antenna far-field calibration. The schematic diagram is shown as figure $1, \Delta \mathrm{r}$ represents the phase change, $\mathrm{D}$ is the diameter of the antenna. 


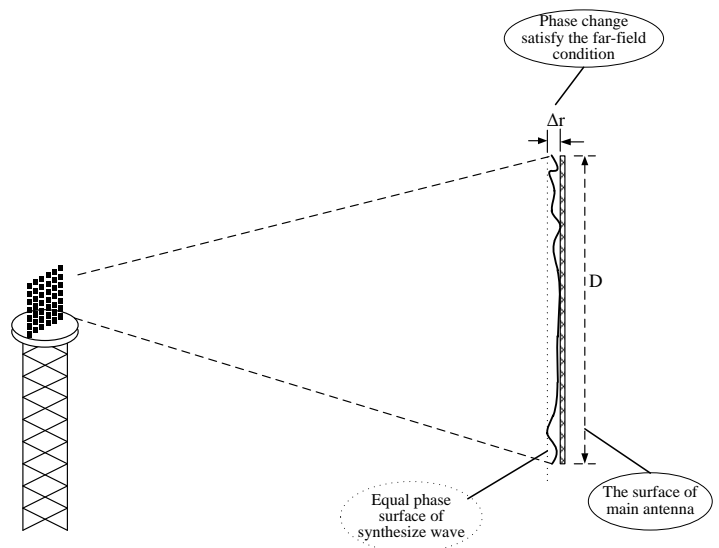

Fig. 1. The signal receiving sketch figure of array antenna

\section{Far-field character analysis of antenna}

\section{A. Basic principle}

Under the condition of far-field distance, spot $\mathrm{P}$ radiate electromagnetic field aimed to area $\mathrm{J}$, watch spot is the coordinate original point $\mathrm{O}, \mathrm{r}$ is path vector from watch point to field point, $r$ is path vector from point $\mathrm{O}$ to the point $\mathrm{Q}$ in the original area, $\alpha$ is the angle of $r$ into $r^{\prime}$, the distance from point $\mathrm{Q}$ to field point $\mathrm{P}$.

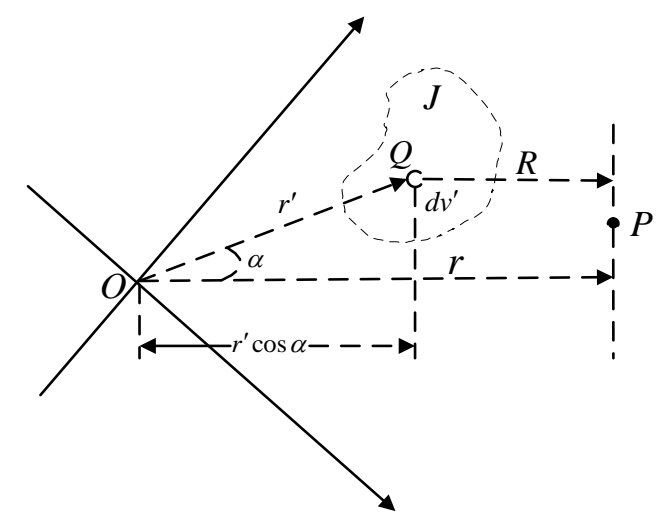

Fig. 2. The parallel approximate figure of the far field calculation

Figure 2 is the parallel approximate figure of the far field calculation, in globe coordinate,

$$
R=r-r^{\prime} \cos \alpha+\frac{1}{r}\left(\frac{r^{\prime 2}}{2} \sin ^{2} \alpha\right)+\frac{1}{r^{2}}\left(\frac{r^{\prime 3}}{x} \cos \alpha \cdot \sin ^{2} \alpha\right)+\cdots
$$

Under the condition of far-field distance, since $r$ is mostly more bigger than $r^{\prime}, r \gg r^{\prime} \gg r^{\prime} \cos \alpha$, thus, $R \approx r-r^{\prime} \cos \alpha(2)$

The formula (2) is called calculation approximate, path vector $\mathrm{R}$ and $r$ can be considered one parallel ray. The deviation due to ignoring the third element of formula (1) is $\lambda / 16^{[1]}$, the corresponding phase difference is : $\frac{2 \pi}{16}=\frac{\pi}{8} \mathrm{rad}=22.5^{\circ}$

\section{B. Index demand of the quasi plane wave}

Something can be seen through the analysis on far-field above, under the condition of far-field distance, the corresponding phase difference is 22.5 degree because of ignoring the third element, meanwhile, the radiate field can be approximately seen as the uniformly plane wave. According to the theory of far-field, the wave formed in the antenna surface region from center to boundary can be approximately seen as quasi 
plane wave under the condition that the phase degree difference between the center and boundary of antenna surface region is less than 22.5 , this region is also called "static region".

According to the different requirements of survey accuracy and process level, the amplitude and phase change requirements to quasi plane wave is also different. The index of references ${ }^{[2]}$ is: the maximum amplitude change in static region is less than $2 \mathrm{~dB}$, the maximum phase change in static region is less than 22.5degree.

\section{Design of antenna array}

The antenna system formed by two more antennas is called multiple element antenna, array antenna is the most common used multiple element antenna. A simple antenna is called antenna unit which is generally weak directional antenna such as horn antenna and slot antenna.

A. Far field model of horn antenna

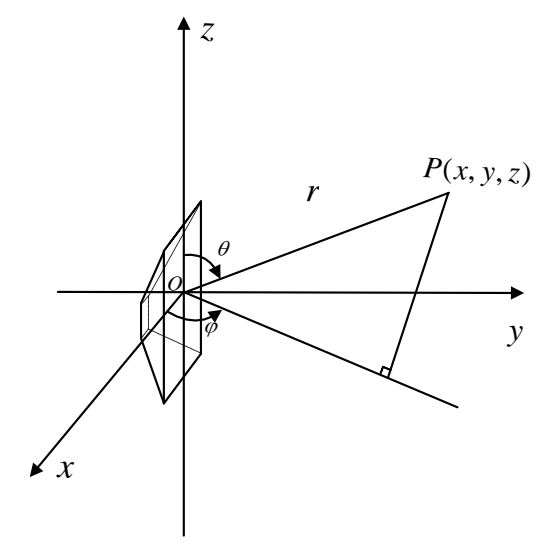

Fig. 3. The far field model of horn antenna

Horn antennas are used to form the 2dimension surface array, we approximately consider that electric current on horn antennas is the line electric current sine distributed as the axis of horn antenna bore when calculating the radiation wave. In figure 3, axis of horn antenna bore is placed on $\mathrm{z}$ axis, center point of horn antenna bore is placed on original point, the watch point is in the far-field area of horn antenna, $r$ is path vector from original point to point $\mathrm{P}, \theta$ is the included angle between path vector $\mathrm{r}$ and $\mathrm{z}$ axis. $\theta$ can be approximately considered 90 degree under condition of far-field, so the far-field model generated by horn antenna on point $\mathrm{P}$ can be expressed as ${ }^{[3]}$ :

$$
\begin{aligned}
& E_{\theta}(P)=-j 60 \frac{I_{0}}{2 \pi} \cos k l \frac{e^{-j k r}}{r} \\
& H_{\varphi}(P)=-j 60 I_{0} \cos k l \frac{e^{-j k r}}{r}
\end{aligned}
$$

\section{B. Superposed field mathematical model of antenna array}

The electromagnetic field of a multi antenna can be applied to the superposition principle, because in general, the electromagnetic field of the antenna is linear with the source. If $\vec{E}_{i}$ is generated by the antenna element, the total field of multiple antenna system made up by $\mathrm{N}$ antenna element is:

$$
\vec{E}=\sum_{i=1}^{N} \vec{E}_{i}
$$

Using $\mathrm{N}^{*} \mathrm{~N}$ two element planar array, as shown in Figure 3, the horn antenna is located in the xoy plane, and the horn is placed along the $\mathrm{X}$ direction. The distance between the array elements along the $\mathrm{X}$ direction 
is $\mathrm{dx}$, and the distance between the array elements is dy along the $\mathrm{Y}$ direction. The distance of the target surface (the observation plane) to the center of the array is $d$. As the target aperture for the radius $R$ of the circular, using polar coordinates representation, $\theta$ is the angle between the line points $\mathrm{P}$ to $\mathrm{O}$ and the $\mathrm{x}$-axis, $\rho$ represents the distance of the observation point $\mathrm{P}$ to $\mathrm{O}_{1}$ point.

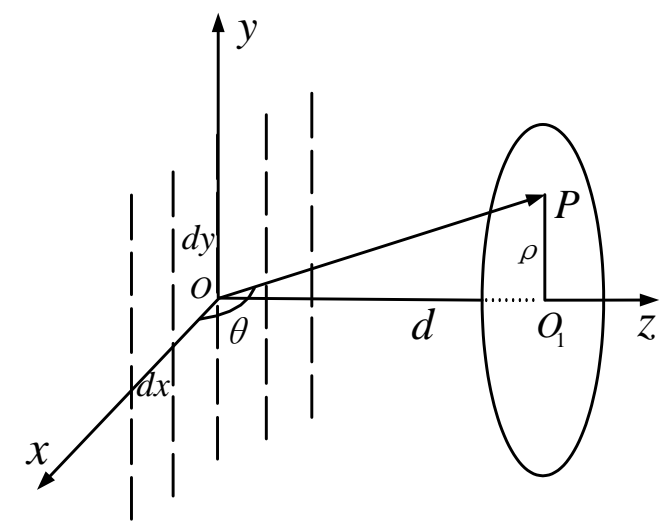

Fig. 4. The schematic diagram of the superposition of antenna array

Set up the distance between a single antenna element $A_{i j}(i=1 、 2 、 \ldots . N$, count from the $X$ axis direction; $\mathrm{j}=1 、 2 、 \ldots . \mathrm{N}$, count from the $\mathrm{Y}$ axis direction)and observation point $P_{\rho \theta}$ is $r_{i j \rho \theta}$, the mathematical model of the superposed d generated by antenna array on observation point $P_{\rho \theta}$ is:

$$
E_{\rho \theta}=\sum_{i=1}^{N} \sum_{j=1}^{N}-j 60 \cos k l \frac{I_{0}}{2 \pi} \frac{e^{-j k r_{i j \rho \theta}}}{r_{i j \rho \theta}}
$$

The above formula is the radiation field formula on the condition which every antenna element parameters is exactly the same. In order to achieve quasi plane wave requirements, we must attach a weight $\mathrm{W}(\mathrm{W}=\mathrm{a} * \mathrm{i}+\mathrm{b} * \mathrm{j})$ to each antenna element, $\mathrm{W}$ contain two aspects of the amplitude and phase which can be achieved through attenuators and phase shifters, so the formula (6) can be changed to:

$$
E_{\rho \theta}=-j 60 \cos k l \frac{I_{0}}{2 \pi} \sum_{i=1}^{N} \sum_{j=1}^{N} W_{i j} \frac{e^{-j k k_{i j \rho \theta}}}{r_{i j \rho \theta}}
$$

\section{Weight optimization of antenna array}

By above knowable, each antenna array element has a weight, and the weight involves two aspects of the amplitude and phase. Only rely on the phase and amplitude constraints of quasi plane wave, it's unable to determine the weights by numerical analysis method. The least square method proposed in the literature ${ }^{[4]}$ is also necessary to determine the ideal field of quasi plane wave, and the result is not the optimal solution. In this paper, the optimization theory is adopted to realize the selection and optimization of the weight $\mathrm{W}_{\mathrm{ij}}$ by the reasonable construction of the objective function and the constraint condition.

The optimization problem with constraints is usually written as:

$$
\begin{aligned}
& \operatorname{minf}(x) \\
& \text { s.t. }\left\{\begin{array}{l}
c_{i}(x)=0, i \in E \\
c_{i}(x) \geq 0, i \in I
\end{array}\right.
\end{aligned}
$$

In the formula (8), I and $\mathrm{E}$ are the index set of equality constraints and inequality constraints, which $\mathrm{cj}(\mathrm{x})$ is the constraint function and $f(x)$ is the objective function. The mixed genetic algorithm based on the thought of genetic algorithm ${ }^{[5]}$ made up by the foundation algorithm function based on Matlab Algorithm Tool and traditional optimization method is used to optimize the result of genetic algorithm. The selection 
and optimization of the weights of $\mathrm{W}_{\mathrm{ij}}$ are realized mainly from two aspects: the analysis of the constraints and the construction of the target function.

According to the requirements of the above quasi plane wave index, on the restrain condition of far field static region which the phase change within 20 degrees, amplitude variation within $0.5 \mathrm{~dB}$. The antenna array weights optimization algorithm can be expressed by formula (9) (11):

$$
\begin{gathered}
f(x)=\max \left\{\left[20 \log a b s\left(E_{\rho \theta}\right)-20 \log a b s\left(E_{00}\right)\right]\right\} \\
c_{1}(x)=0.5-\max \left\{\left[20 \log a b s\left(E_{\rho \theta}\right)-20 \log a b s\left(E_{00}\right)\right]\right\} \\
c_{2}(x)=20-\max \left[\operatorname{angle}\left(E_{\rho \theta}\right)\right]
\end{gathered}
$$

Among them, $\rho=0 \sim D / 2, \theta=0 \sim 2 \pi, \mathrm{f}(\mathrm{x})$ is the maximum phase difference between the center and the boundary of the observation surface, $\mathrm{c}_{1}(\mathrm{x})$ is the constraint condition of the amplitude change, $\mathrm{c}_{2}(\mathrm{x})$ is the constraint condition of the phase change. Compared to the least squares method in literature 4 ,comprehensive using genetic algorithm to optimize the weights, the realization is simple, and the weight is more optimized and reasonable.

\section{Computer simulation}

According to the above theory, setting a suitable frequency, the distance between calibration tower and the measured antenna is 1000 meters, antenna array is a two-dimensional planar array made up by $4 * 416$ horn antennas, antenna diameter D is 12 meters. Through simulation, when the horn antenna spacing design is 0.968 meter, antenna array synthesis wave can better satisfy the quasi plane wave conditions. According to the mathematical model of the horn antenna and the principle of superposition field, we can obtain the 3D simulation results of the amplitude and phase of the synthesized static region processed by optimized algorithm, as shown in Figure 5 and figure 6.

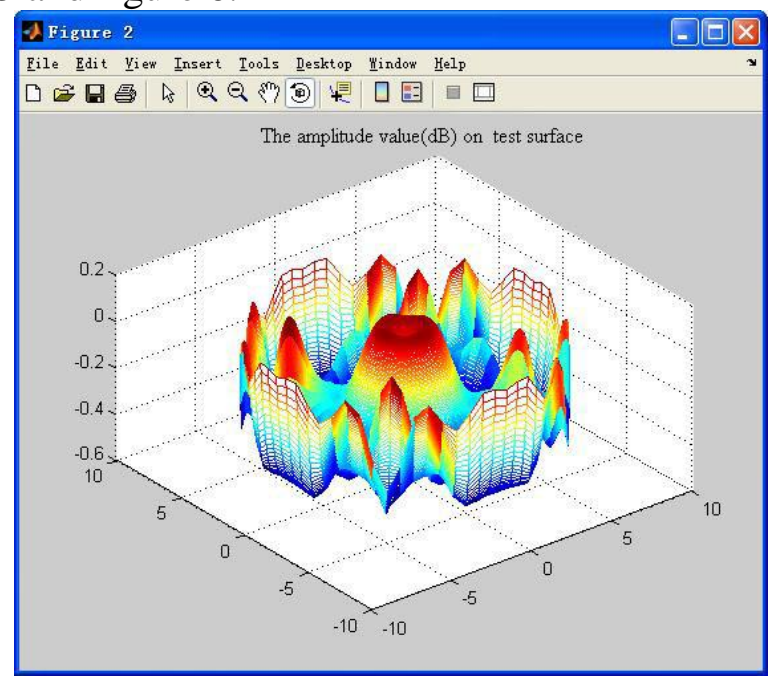

Fig. 5. The $3 \mathrm{~d}$ amplitude simulation results figure in quiet zone 


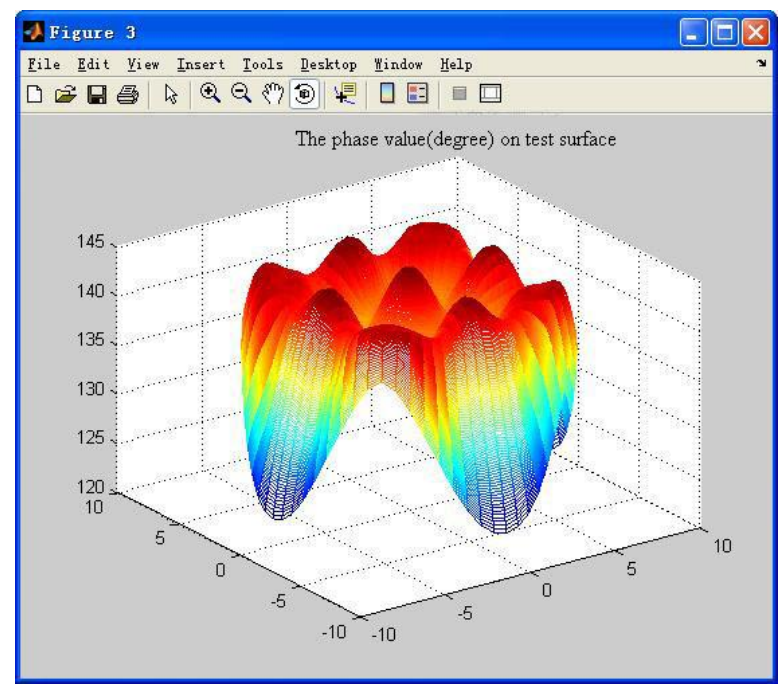

Fig. 6. The $3 \mathrm{~d}$ phase simulation results figure in quiet zone

The $2 \mathrm{~d}$ phase simulation results figure on $\mathrm{X}$ axis can be got through the further phase change data processing, it's shown as the figure 7 .

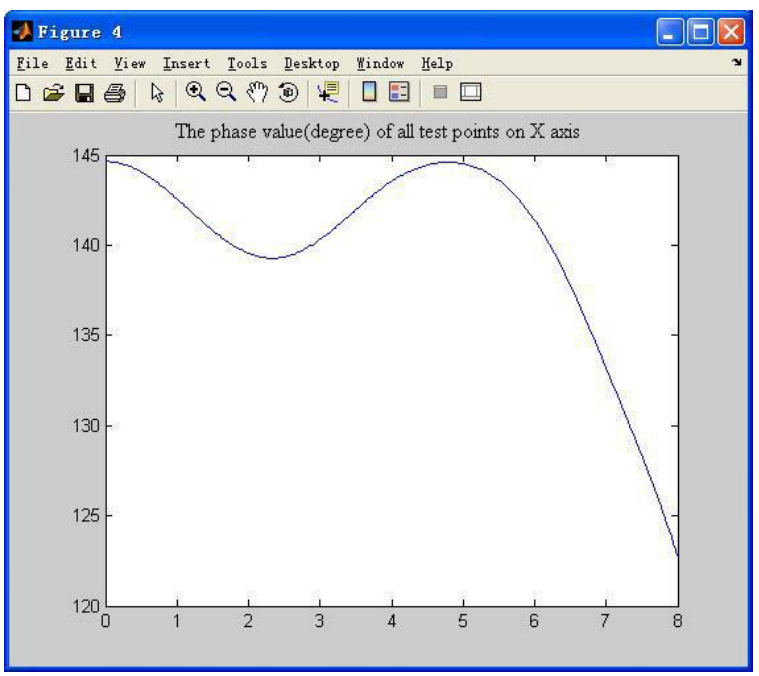

Fig. 7. The $2 \mathrm{~d}$ phase simulation results figure on $\mathrm{X}$ axis

From the figure 5、6、7, it can be seen that the maximum variation of the plane wave from the origin to the boundary $\Delta \beta$ is $0.508 \mathrm{~dB}<2 \mathrm{~dB}$, and the maximum phase difference $\Delta \Phi=21.93^{\circ}<22.5^{\circ}$ between the original point and the boundary which $\mathrm{x}$ is 8 meters. The amplitude and phase data meet the requirements of the quasi plane wave. In the center of the coordinate origin, a static area with the diameter of 16 meter is obtained by simulation which can effectively solve the problem of the far field calibration of the antenna with a diameter of $12 \mathrm{~m} \mathrm{X}$ band.

\section{Testsituation}

A. Test prepare

In view of the limitations of cost and site conditions, the method of $3 * 3$ array antenna is used to test the proposed method. Procurement of the relevant test equipment, the main has a removable array antenna bracket, one array antenna base, 9X band line polarization horn, 12 cables of 6 meters low loss, one self-developed network of power division and phase shift. 


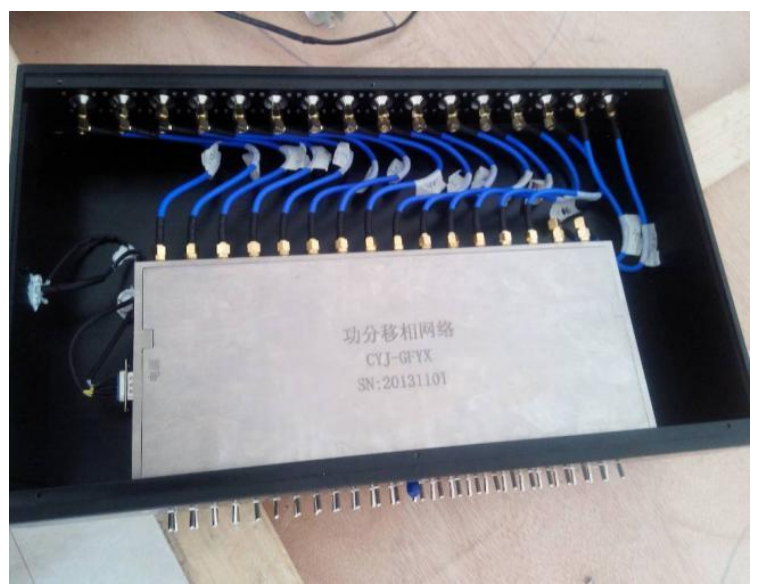

Fig. 8. Figure 8 The real figure of the network of power division and phase shift

The network of power division and phase shift is shown in Figure 8, with one signal input and 16 signal output, with a phase control switch and a gain control switch. The phase and gain control is composed of a set of two rows. Twenty four phase control switches beside in first row, consist of four groups (each group of six control code, a total of 64 States) 2 phase control code, phase control accuracy is: 360/64=5.6 degrees; twenty four amplitude control switches beside in second row, consist of four groups (each group of six control code, a total of 64 States) 2 amplitude control code, each amplitude control accuracy is $0.5 \mathrm{~dB}$, amplitude control range $32 \mathrm{~dB}$.

\section{B. Experiment test}

According to the requirements of the quasi plane wave, the signal amplitude from the center to the boundary of the antenna is less than $2 \mathrm{~dB}$, and the phase change should be less than 22.5 degrees. In order to ensure the test results, RF output signal amplitude difference of the network of power division and phase shift should be less than $0.5 \mathrm{~dB}$, phase difference should be less than 5 degrees under the condition that phase shifting control switch and the gain control switch are arranged to zero.

Measured by the first output signal, the phase and amplitude data of the other port output signals are shown as table I. The amplitude data of each port is less than $0.4 \mathrm{~dB}$ and the phase difference is less than 4.6 degrees.

As electric length of the cable under different condition of the straight and bending will change, resulting in phase change of the cable. The less phase change of the cable change, the better the states of cable has. The phase change values under two conditions of natural extension and natural bending are tested to find the phase change characteristics of the cable are tested, results are shown in table II:

The phase difference change of nine cables: number $1,3,4,5,6,7,8,10,11$ under two conditions of natural extension and natural bending is relatively little which can be used to experiment test.

\section{Experiment result and analysis}

In order to test whether the electromagnetic wave generated by the field test has the characteristics of quasi plane wave, the antenna array antenna and a single horn antenna are arranged 2450 meters far away from the antenna of UXB system, a horn antenna is arranged 9000 meters far away from the antenna. The antenna array is positioned to face the surface of UXB antenna. The signal source is used to send out the radio frequency signal, the phase and gain control switch of the network of power division and phase shift can be adjusted so that the electromagnetic wave of the array antenna can meet the requirements of the quasi plane wave. Install physical and test as shown in Figure 9 and figure 10. 


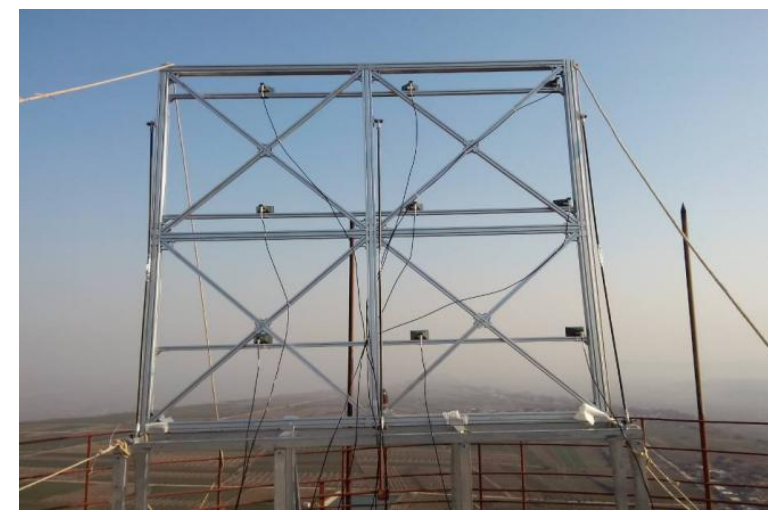

Fig. 9. The install physical figure of array antenna

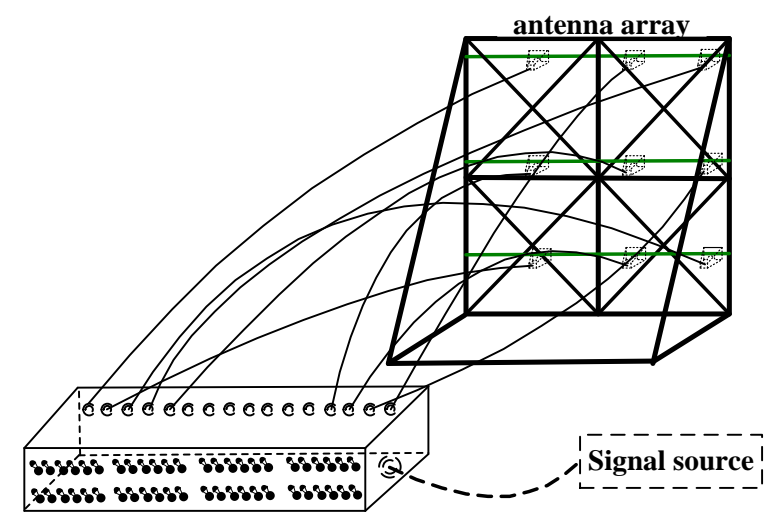

Fig. 10. The test figure of array antenna

The directional sensitivity and cross coupling are tested aimed at three conditions that the near-field array antenna near-field single horn antenna and far-field single horn beacon, the results are shown in figure 11 and figure 12.

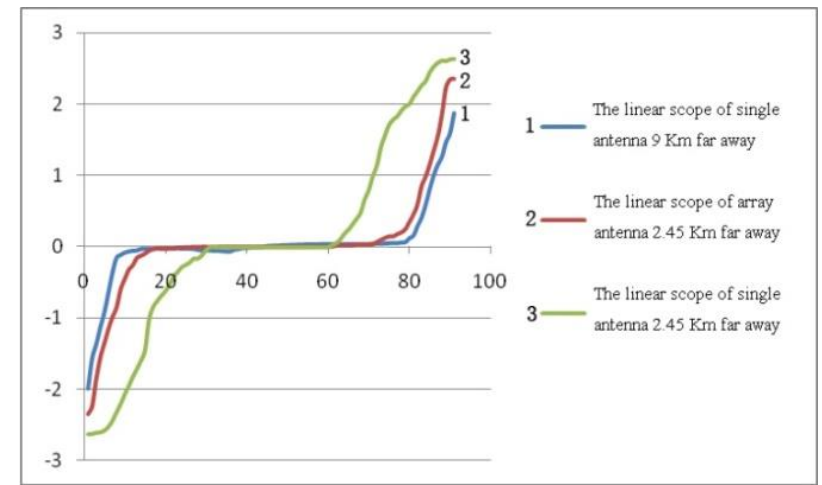

Fig. 11. The linear contrast figure of bearing error voltage in different conditions 


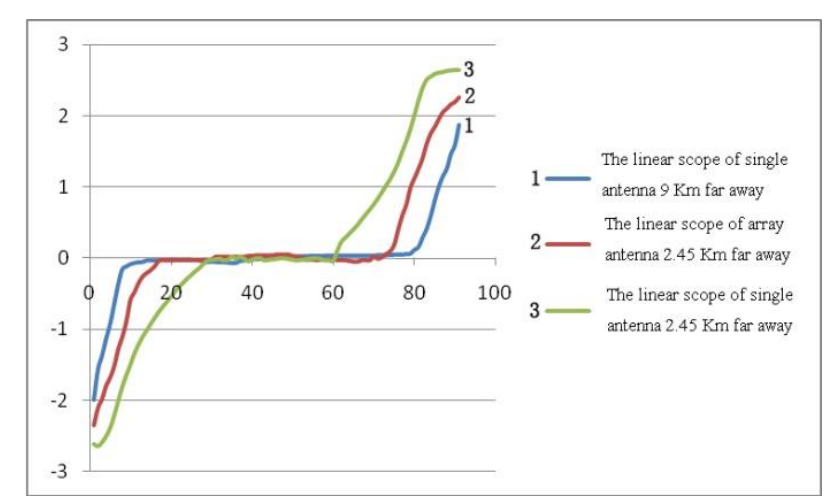

Fig. 12. The linear contrast figure of pitch error voltage in different conditions

Figure 11 and 10 show the linear contrast of bearing and pitching error voltage under different conditions. Vertical coordinates express the error voltage size, unit: $\mathrm{V}$, horizontal coordinates express the number of points, no measurement unit, take a point in the antenna pull process every $50 \mathrm{~ms}$, a total of 90 points. The results show that: the data obtained through the test of directional sensitivity and cross coupling under the condition of near field are similar to the results obtained by using the single horn antenna under the far-field condition. Hence, the way put forward by this paper can basically satisfy the requirement of far-field calibration.

\section{Concluding}

The calibration of the tower is the most stable and reliable calibration method. With the increase of the frequency of UXB system, the distance from the standard calibration tower can't meet the requirements of the far field calibration. The antenna arrays are innovatively used to tower calibration in this paper, the method proposed by using antenna array to synthetic quasi plane wave has passed the physical installation and tower test, basically solved the problem that UXB antenna on survey ship has no tower to calibrate under the near field conditions, so the method has high practical value. But in the process of testing, the accuracy of the antenna array is high, the control accuracy of $4 * 4$ array antenna is limited. Next, the modular surface of antenna array will be used to ensure the accuracy of the antenna array, at the same time, the numbers of antenna arrays will be also increased to improve the accuracy of the antenna array.

\section{References}

[1]KANG Xingjian. Antenna Principle and Design [M]. Beijing Institute of Technology Press, 1993: 20-30.

[2]YANG Yangjiong. Study of Array Antenna and Millimeter-Wave CATR Antenna [D]. Xian: Xian Electronic Science \& Technology University, in April 2011.

[3]XIAN Chufang. Antenna Principles [M]. Beijing Institute of Technology Press, 1993: 20-30.

[4]HE Lei.Plane Wave Synthesis Theory and Application [D].Xian : Xian Electronic Science \& Technology University, in February 2006.

[5]ZHANG Wang. Analysis on Synthesis of Array Antenna Based on Genetic Algorithm and its Key Points. [J]. Antenna and servo technology, 2011, 37(4): 28-32. 
TABLE I. THE TEST RESUlTS OF PHASE SHIFT NETWORK

\begin{tabular}{|c|c|c|c|c|c|c|c|c|}
\hline Port & 1 & 2 & 3 & 4 & 5 & 6 & 7 & 8 \\
\hline $\begin{array}{c}\text { Signal } \\
\text { Phase difference } \\
\text { (degree) }\end{array}$ & 0 & 0.7 & -2.3 & -2.5 & -2.9 & -0.8 & -1.2 & -2.3 \\
\hline $\begin{array}{c}\text { Amplitude } \\
\text { difference (dB) }\end{array}$ & 0 & 0.2 & 0.01 & 0.00 & 0.2 & 0.06 & $\begin{array}{c}-0.0 \\
2\end{array}$ & 0.06 \\
\hline Pignal Port & 9 & 10 & 11 & 12 & 13 & 14 & 15 & 16 \\
\hline $\begin{array}{c}\text { Phase difference } \\
\text { (degree) }\end{array}$ & 0.6 & -3.0 & -0.7 & -1.3 & -3.0 & -4.5 & -4.6 & -0.2 \\
\hline $\begin{array}{c}\text { Amplitude } \\
\text { difference (dB) }\end{array}$ & 0.0 & -0.2 & -0.2 & -0.4 & 0.02 & 0.1 & 0.2 & 0 \\
\hline
\end{tabular}

TABLE II. PHASE TEST RESULTS OF THE CONNECTING CABLE IN DIFFERENT CONDITIONS

\begin{tabular}{|l|c|c|c|c|c|c|}
\hline Cable number & 1 & 2 & 3 & 4 & 5 & 6 \\
\hline $\begin{array}{l}\text { Phase difference under natural status } \\
\text { extension condition (degree) }\end{array}$ & -1 & 14 & 31 & 17 & -1 & 9 \\
\hline $\begin{array}{l}\text { Phase difference under natural bending } \\
\text { condition (degree) }\end{array}$ & 14 & 32 & 42 & 12 & 11 & 24 \\
\hline $\begin{array}{l}\text { Phase difference under two different } \\
\text { conditions (degree) }\end{array}$ & 15 & 18 & 11 & -5 & 12 & 15 \\
\hline $\begin{array}{l}\text { Signal status Cable number } \\
\text { Phase difference under natural } \\
\text { extension condition (degree) }\end{array}$ & 7 & 8 & 9 & 10 & 11 & 12 \\
\hline $\begin{array}{l}\text { Phase difference under natural bending } \\
\text { condition (degree) }\end{array}$ & 23 & 20 & 38 & 25 & 28 & 39 \\
\hline $\begin{array}{l}\text { Phase difference under two different } \\
\text { conditions (degree) }\end{array}$ & 16 & 8 & 17 & 13 & 3 & 22 \\
\hline
\end{tabular}

